onkopedia

onkopedia leitlinien

\title{
Mammakarzinom des Mannes
}

\section{Leitlinie}

ICD10: C50.9

Empfehlungen der Fachgesellschaft zur Diagnostik und Therapie hämatologischer und onkologischer Erkrankungen

\section{DGHO: OeGHO Şక్షMO}




\section{Herausgeber}

DGHO Deutsche Gesellschaft für Hämatologie und Medizinische Onkologie e.V.

Alexanderplatz 1

10178 Berlin

Geschäftsführender Vorsitzender: Prof. Dr. med. Carsten Bokemeyer

Telefon: $+49(0) 3027876089-0$

Telefax: +49(0)3027876089-18

info@dgho.de

www.dgho.de

\section{Ansprechpartner}

Prof. Dr. med. Bernhard Wörmann

Medizinischer Leiter

\section{Quelle}

www.onkopedia.com

Die Empfehlungen der DGHO für die Diagnostik und Therapie hämatologischer und onkologischer Erkrankungen entbinden die verantwortliche Ärztin / den verantwortlichen Arzt nicht davon, notwendige Diagnostik, Indikationen, Kontraindikationen und Dosierungen im Einzelfall zu überprüfen! Die DGHO übernimmt für Empfehlungen keine Gewähr. 


\section{Inhaltsverzeichnis}

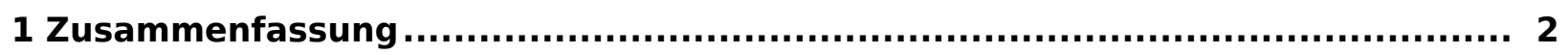

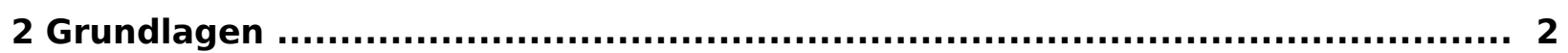

2.1 Definition und Basisinformation ....................................................................... 2

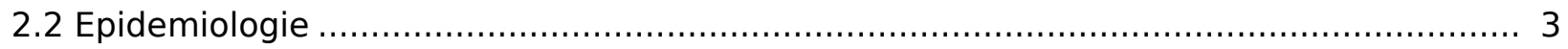

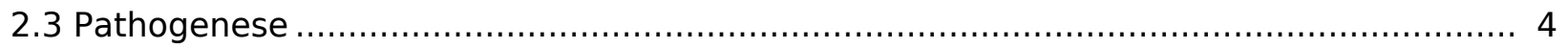

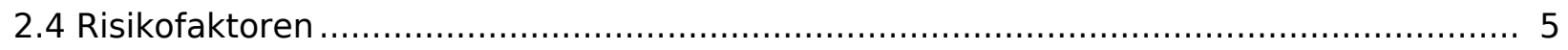

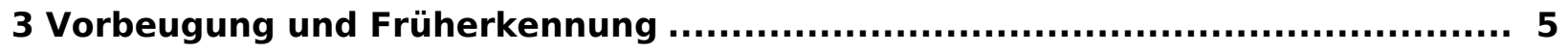

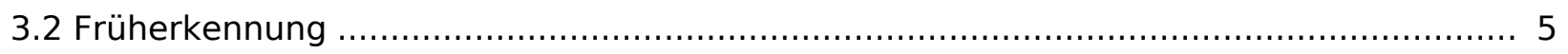

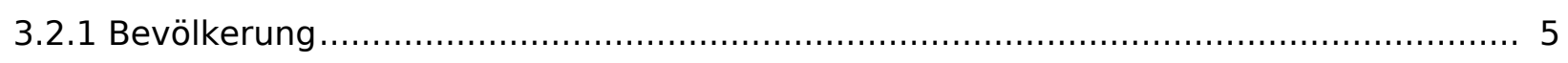

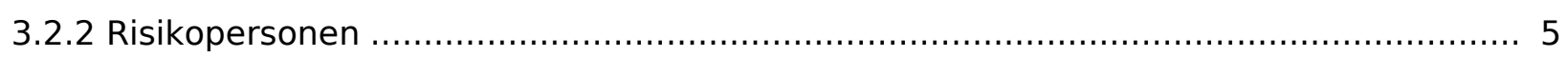

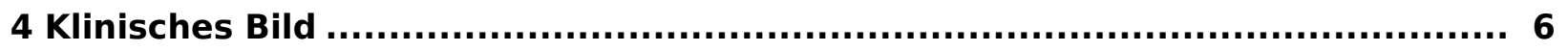

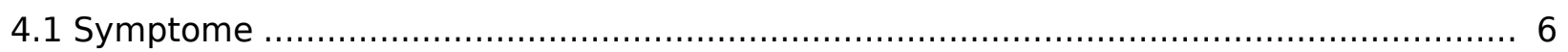

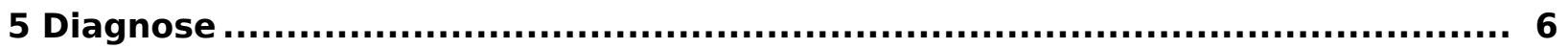

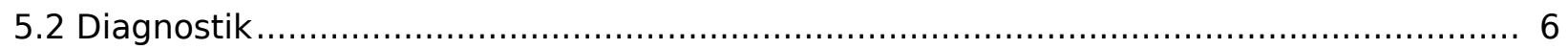

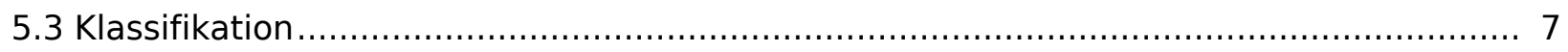

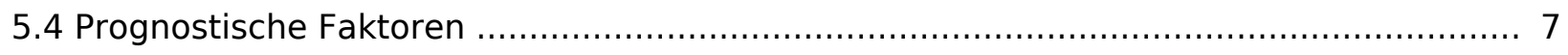

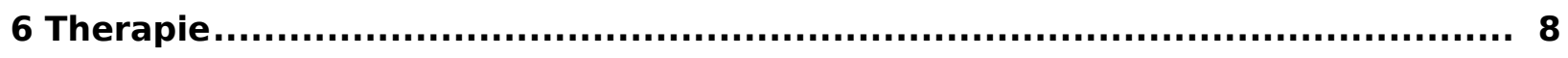

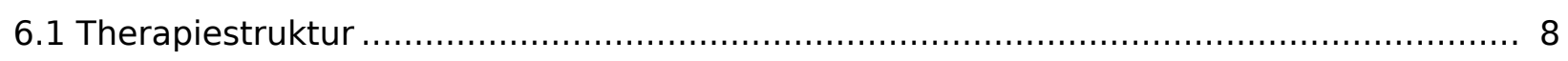

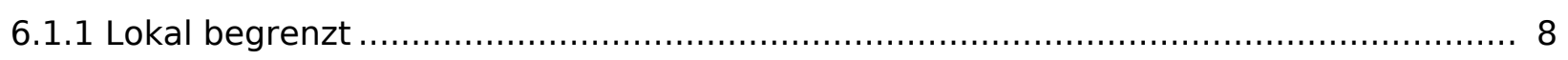

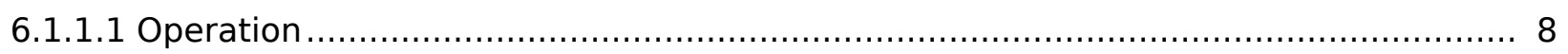

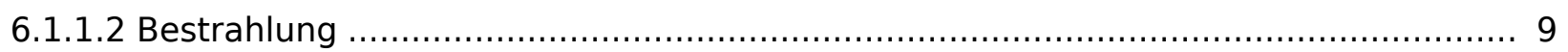

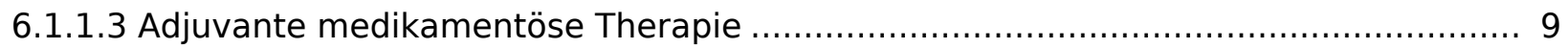

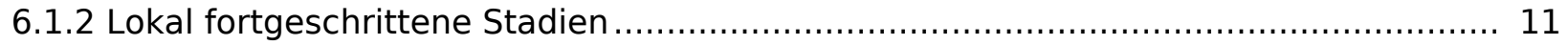

6.1.2.1 Primäre (neoadjuvante) medikamentöse Therapie ......................................... 11

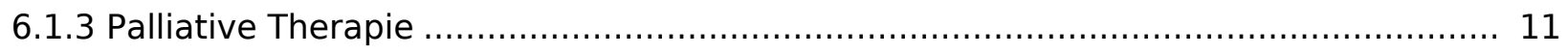

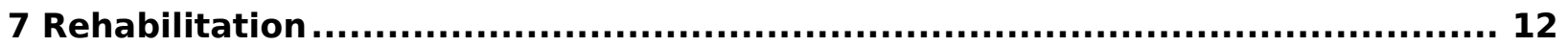

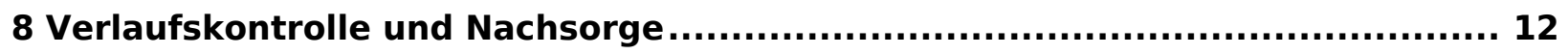

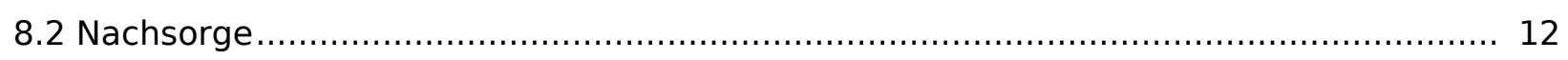

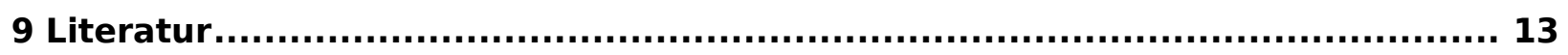

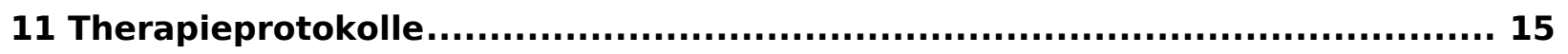

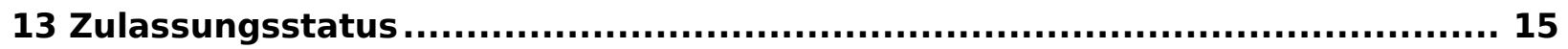

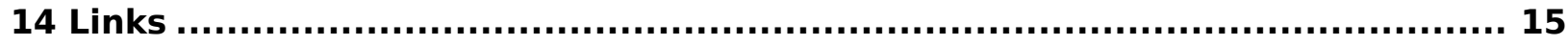

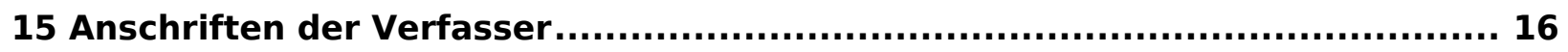

16 Erklärungen zu möglichen Interessenkonflikten .............................. 18 


\section{Mammakarzinom des Mannes}

ICD10: C50.9

Stand: August 2016

Autoren: Bernhard Wörmann, Stefan Aebi, Marija Balic, Thomas Decker, Tanja Fehm, Richard Greil, Nadia Harbeck, Barbara Krug, Friedrich Overkamp, Oliver Rick, Frederik Wenz, Diana Lüftner

Autoren früherer Versionen: Kurt Possinger

\section{Zusammenfassung}

Das Mammakarzinom ist bei Männern eine seltene Erkrankung. Etwa ein Viertel der Patienten hat eine genetische Prädisposition, am häufigsten mit BRCA2-Mutationen, der CHEK2Mutation del1100C oder dem Klinefelter-Syndrom. Histologisch dominiert das invasive duktale Karzinom. Die meisten Erfahrungen in Diagnostik und Therapie des Mammakarzinoms wurden bei Frauen gesammelt und auf die Situation der Männer übertragen. Die Prognose wird vor allem vom Stadium bei Erstdiagnose und von der Biologie der Erkrankung bestimmt.

Der Therapieanspruch ist kurativ im lokal begrenzten, im lokal fortgeschrittenen und im lokal rezidivierten Stadium. Die Therapie ist in diesen Krankheitsstadien multimodal. Nach Operation und Bestrahlung schließt sich bei den meisten Patienten eine adjuvante endokrine Therapie mit Tamoxifen an, ggf. auch eine Chemo- und/oder eine anti-HER2-Therapie. Im Stadium der Fernmetastasierung ist der Therapieanspruch palliativ mit dem Ziel der Linderung von Symptomen und Verlängerung der Überlebenszeit.

Die relative 5-Jahres-Überlebensrate liegt in Deutschland bei 74\%, niedriger als bei Frauen mit Mammakarzinom.

\section{Grundlagen}

\subsection{Definition und Basisinformation}

Die Verteilung der histologischen und der immunhistologischen Befunde unterscheidet sich beim Mammakarzinom des Mannes (MBC (Male Breast Cancer) deutlich von den Frauen. Histologisch wird bei etwa $90 \%$ der männlichen Patienten ein invasiv duktales Karzinom gefunden. Das duktale Carcinoma in situ (DCIS) macht 5-10\% der Diagnosen bei klinischem und/oder bildgebendem Verdacht auf ein Mammakarzinom aus [20]. Andere histologische Subtypen sind selten. Bei über $90 \%$ der Karzinome ist die Östrogen (ER)-Expression hoch (Allred 7-8) [9, 20]. Bei über 90\% ist der Progesteron (PR)-Rezeptor nachweisbar, aber nur in etwa 35\% hoch exprimiert. HER2-positive Karzinome machen 5-10\% aus, triple negative Karzinome sind selten. Eine hohe Expression des Androgenrezeptors ist bei $85-90 \%$ der Karzinome nachweisbar [36]. Das histologische Grading wird bei 50-55\% der Karzinome als G2 eingestuft [Fentiman, Cardoso]. Männer mit Mammakarzinom haben in etwa 35-40\% einen positiven Lymphknotenstatus [24]. 


\subsection{Epidemiologie}

In Deutschland erkranken pro Jahr etwa 600 [23], in Österreich erkrankten im Jahr 201273 [35], in der Schweiz zwischen 1987 und 2011 durchschnittlich 32 Männer/Jahr an Brustkrebs [31]. Dies entspricht in Deutschland einer rohen Erkrankungsrate von 1,5/100.000 Männer und altersstandardisiert von 1/100.000. Das mittlere Erkrankungsalter liegt bei 71 Jahren (bezogen auf Zeitraum von 2009-2013), siehe Abbildung 1.

\section{Abbildung 1: Inzidenz und Mortalität des Mammakarzinoms bei Männern in Deutschland}
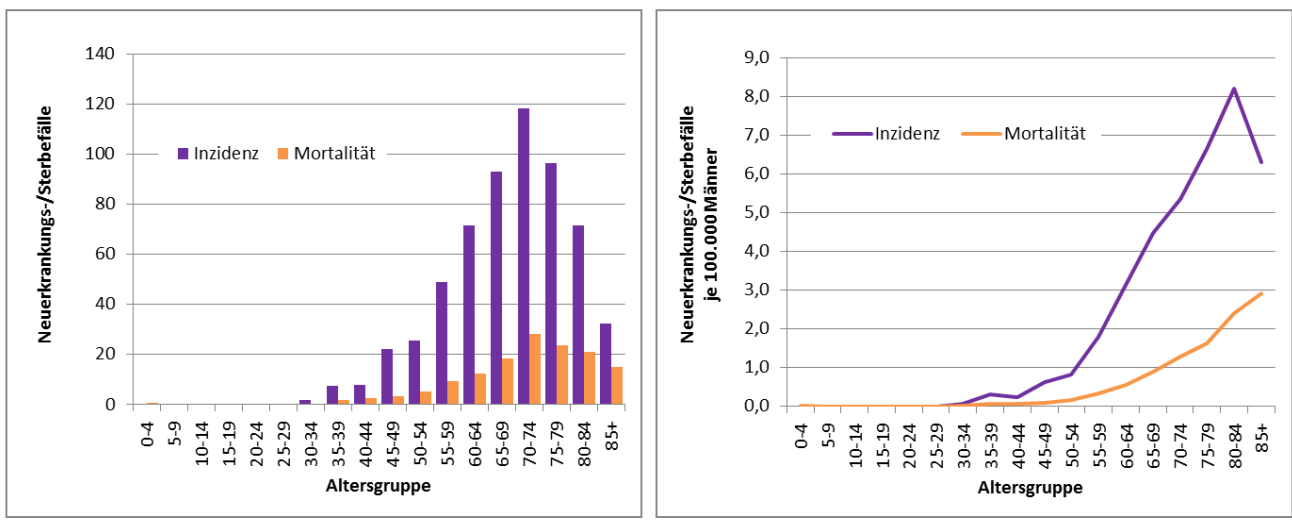

Legende:

Altersspezifische Inzidenz und Mortalität des Brustkrebses bei Männern in Deutschland als Fallzahl (links) und als Rate (rechts)

Berechnungen und Grafik: Institut für Krebsepidemiologie, Quellen: GEKID-Atlas (Inzidenz), Statistisches Bundesamt, Todesursachenstatistik (Mortalität) International schwankt die Inzidenz des männlichen Mammakarzinoms erheblich zwischen den verschiedenen Regionen und Nationen [30].

International schwankt die Inzidenz des männlichen Mammakarzinoms erheblich zwischen den verschiedenen Regionen und Nationen [30].

Jährlich sterben in Deutschland durchschnittlich (2009-2013) etwa 140 Männer an Brustkrebs (Datenquelle: Statistisches Bundesamt, Todesursachenstatistik). Dies entspricht einer rohen Rate von 0,4 Todesfällen pro 100.000 (Altersstandardisierte Rate (Europastandard): 0,2 pro 100.000). Das mediane Sterbealter liegt bei 73 Jahren. Im zeitlichen Verlauf deutet sich ein leichter Anstieg der Inzidenz bei gleichzeitig leichtem Rückgang der Mortalität an, siehe Abbildung 2. Allerdings ist der männliche Brustkrebs verhältnismäßig selten. Die Daten sind daher mit Unsicherheiten und entsprechenden Schwankungen behaftet.

Abbildung 2: Zeitliche Entwicklung der Brustkrebsinzidenz und -mortalität bei Männern in Deutschland
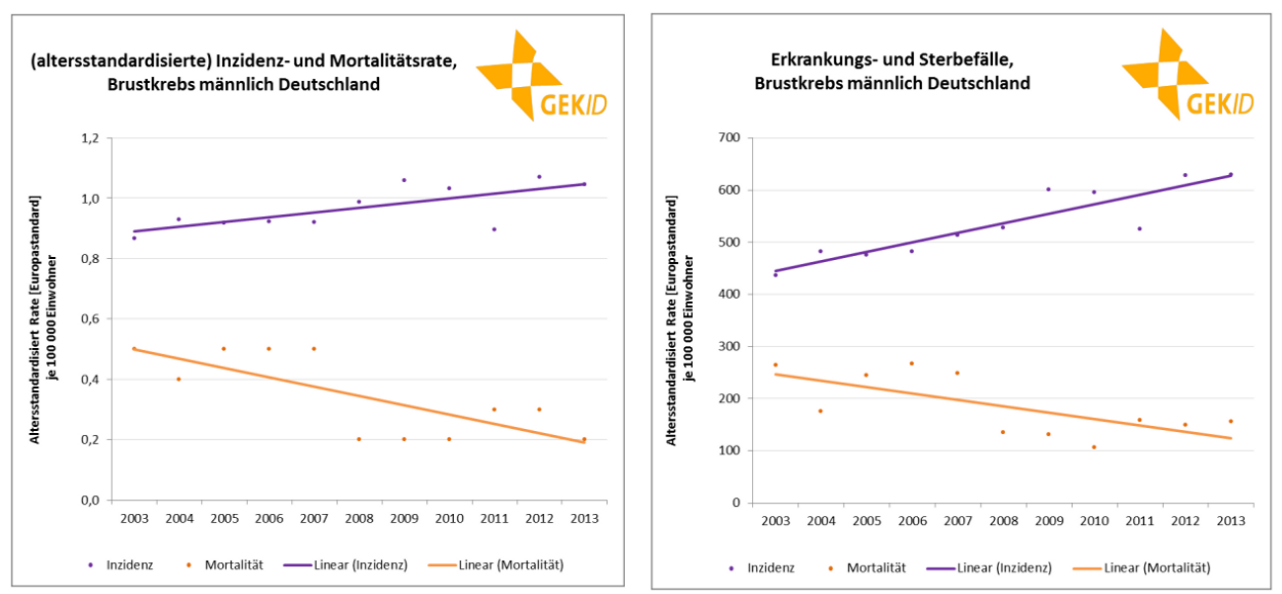
Im Vergleich zum weiblichen Brustkrebs ist der männliche Brustkrebs sehr selten. Je 117 erstmalig erkrankten Frauen wurde ein erkrankter Mann diagnostiziert (2009-2013). Hinsichtlich des Erkrankungsalters unterscheiden sich beide Geschlechter deutlich voneinander, siehe Abbildung 3.

Abbildung 3: Vergleich der Altersverteilung bei Inzidenz und Mortalität zwischen den Geschlechtern beim Brustkrebs in Deutschland 2009 - 2013
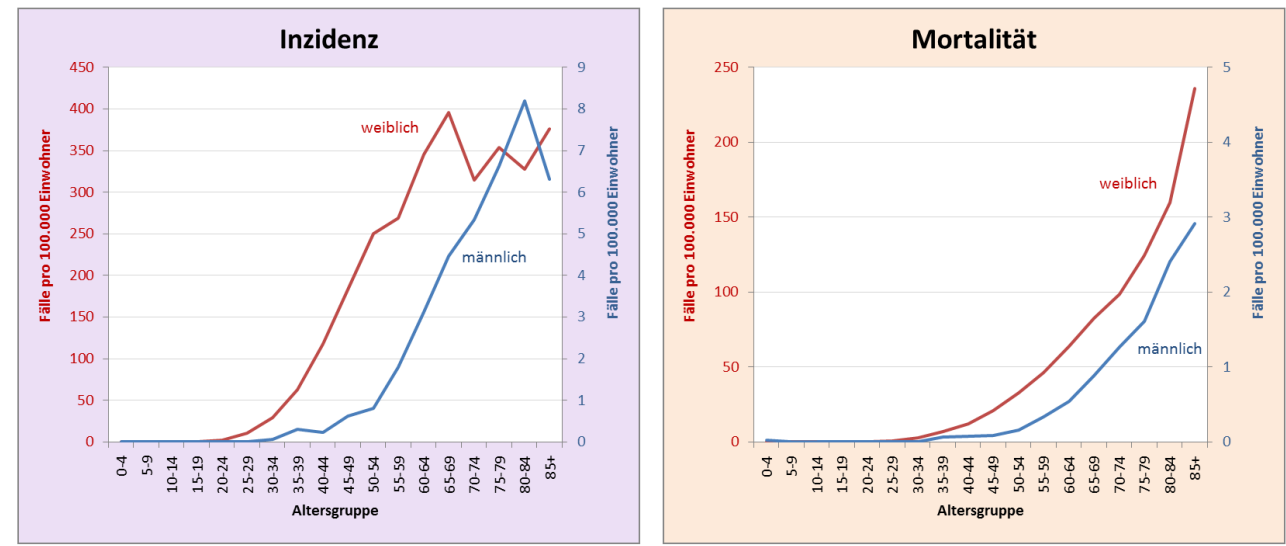

\section{Legende:}

Wegen der unterschiedlichen Häufigkeit zwischen den Geschlechtern sind die Werte für Frauen auf der linken Vertikalachse angegeben[rot] und für Männer auf der rechten [blau].)

Berechnungen und Grafik: Institut für Krebsepidemiologie, Quellen: GEKID-Atlas (Inzidenz), Statistisches Bundesamt, Todesursachenstatistik (Mortalität)

Vernachlässigt man den zahlenmäßigen Unterschied, zeigt sich, dass der starke Anstieg des Erkrankungsrisikos bei Frauen etwa 20 Jahre vor dem Anstieg bei Männern liegt. Der Altersgipfel liegt bei Frauen zwischen 65 und 69 Jahren, bei Männern hingegen erst zwischen 80 und 84 Jahren. Etwas geringer ist der Unterschied bei der Mortalität. Das zeigt sich auch am durchschnittlichen Erkrankungs- und Sterbealter. Frauen erkranken im Median mit 64 Jahren, Männer mit 71 Jahren. Das mediane Sterbealter liegt bei beiden Geschlechtern bei 73 Jahren. Weitere Angaben zum zeitlichen Verlauf, zur regionalen Verteilung und altersspezifische Angaben im Zeitverlauf findet man unter http://www.gekid.de/Atlas/CurrentVersion/ atlas.html.

\subsection{Pathogenese}

Die Ätiologie des männlichen Mammakarzinoms ist nicht geklärt. Der Anteil von Patienten mit molekularbiologisch charakterisierter, genetischer Prädisposition liegt deutlich höher als beim Mammakarzinom der Frau.

Eine besondere Rolle in der Pathogenese spielt möglicherweise die Verschiebung des Androgen/Östrogen-Verhältnisses oder ein erhöhter Östradiol-Spiegel [7,8]. Diese hormonelle Veränderung kann zum erhöhten Risiko bei Männern mit Klinefelter-Syndrom, bei Männern mit Hypogonadismus anderer Genese und bei Patienten mit chronischer Leberinsuffizienz beitragen.

Eine eigene Rolle spielt der Androgenrezeptor (AR). Eine hohe AR-Expression auf den Tumorzellen korreliert mit einem höheren Risiko für Lymphknotenmetastasen bei Erstdiagnose und ist mit einer ungünstigen Prognose assoziiert [36]. 


\subsection{Risikofaktoren}

Als Risikofaktoren wurden identifiziert [6, 21]:

- genetisch

- Klinefelter-Syndrom (47, XXY): etwa 20-60fach erhöhtes Erkrankungsrisiko gegenüber der Normalbevölkerung, macht etwa 7\% aller MBC aus, mittleres Erkrankungsalter 58 Jahre; siehe Onkopedia Klinefelter Syndrom

- BRCA2-Mutation: etwa 80fach erhöhtes Erkrankungsrisiko gegenüber der Normalbevölkerung, 5-10\% Erkrankungsrisiko bei männlichen Trägern von BRCA2-Mutationen, macht etwa $10 \%$ bei unselektionierten MBC aus, mittleres Erkrankungsalter 55-60 Jahre; [15, 34]

- BRCA1-Mutation: etwas erhöhtes Erkrankungsrisiko gegenüber Normalbevölkerung, $<2 \%$ bei unselektionierten $M B C$, möglicherweise erhöhtes Risiko für bestimmte Mutationen; [15, 34]

- CHEK2 1100delC-Mutation: etwa 10fach erhöhtes Erkrankungsrisiko gegenüber der Normalbevölkerung, 9\% aller MBC, Nord-Süd-Gefälle in Europa; [11]

- positive Familienanamnese: etwa 2,5 fach erhöhtes Risiko

- erworben

- Kryptorchismus

- Z. n. Orchitis / Epididymitis

- Adipositas, hoher Body-Mass-Index [6, 7]

- Gynäkomastie [Brinton 2010]

- toxisch

- Strahlenexposition der Brustwand

Eine Assoziation mit anderen Risikofaktoren wie hohem Alkoholkonsum oder chronischer Lebererkrankung wurde ebenfalls beschrieben [21], die Bewertung der epidemiologischen Daten ist aber nicht einheitlich.

\section{Vorbeugung und Früherkennung}

\subsection{Früherkennung}

\subsubsection{Bevölkerung}

Es gibt kein Früherkennungsprogramm wie bei Frauen.

\subsubsection{Risikopersonen}

In Familien mit BRCA2-Mutationen wird eine genetische Beratung auch für die Männer empfohlen. Das Klinefelter-Syndrom ist mit einem 20-60fach erhöhten Risiko für ein MBC belastet. Für diese Risikogruppe erscheint ein Früherkennungsprogramm sinnvoll, ist aber nicht implementiert, siehe Onkopedia Klinefelter-Syndrom. 


\section{Klinisches Bild}

\subsection{Symptome}

Dominierendes Symptom ist die schmerzlose Knotenbildung in der Brust, am häufigsten retroareolär. Weitere lokale Symptome sind Hautveränderungen über dem Tumor und Veränderungen der Mamille mit Einziehung, Ulzeration oder Sekretion. Allgemeinsymptome fehlen in frühen Stadien. In fortgeschrittenen Stadien kann es zu Gewichtsabnahme und Leistungsminderung kommen. Symptome aufgrund von Metastasen sind Schwellung des Arms durch Lymphödem bei Lymphknotenmetastasen der Axilla, Knochenschmerzen bei Skelettmetastasen, Husten und Dyspnoe bei pulmonaler und/oder pleuraler Metastasierung, Ikterus und Leberinsuffizienz bei fortgeschrittener Lebermetastasierung oder neurologische Symptome bei zerebraler Metastasierung.

\section{Diagnose}

\subsection{Diagnostik}

Sorgfältige Anamnese und komplette körperliche Untersuchung sind Grundlage rationeller Diagnostik. Der erste Schritt ist die Bestätigung der klinischen und/oder bildgebenden Verdachtsdiagnose, s. Tabelle 1.

Tabelle 1: Diagnostik bei neu aufgetretenen Symptomen [10]

\begin{tabular}{|l|l|}
\hline Untersuchung & Anmerkung \\
\hline Mammographie bds. & Methode der ersten Wahl \\
\hline $\begin{array}{l}\text { Sonographie beider Mammae und der Axillae } \\
\text { Bestätigung der mammographischen Verdachtsdiagnose und Charakterisie- } \\
\text { rung des Befundes }\end{array}$ \\
\hline $\begin{array}{l}\text { Biopsie (Stanzbiopsie, Vakuumbiopsie oder } \\
\text { offene Biopsie) }\end{array}$ & wegen des hohen Risikos der Thoraxwandinfiltration \\
\hline $\begin{array}{l}\text { Magnetresonanztomographie bds. mit Kon- } \\
\text { trastmittel }\end{array}$ & \\
\hline
\end{tabular}

Nach Diagnose eines Mammakarzinoms ist eine gezielte Ausbreitungsdiagnostik (Staging) für Patienten mit Tumoren $\geq \mathrm{pT} 2$ und bei klinischen Symptomen indiziert, siehe Tabelle 2. Fernmetastasen können beim Mammakarzinom in fast allen Regionen des Körpers auftreten. Die häufigsten Lokalisationen sind Skelett, Leber und Lunge.

Tabelle 2: Ausbreitungsdiagnostik

\begin{tabular}{|l|l|l|}
\hline Verdacht & Untersuchung (1. Wahl) & Untersuchung zur Bestätigung / bei Unklarheit \\
\hline Thoraxwandinfiltration & Magnetresonanztomographie mit Kontrastmittel & \\
\hline Skelettmetastasen & Skelettszintigraphie & Röntgen, MRT \\
\hline Lebermetastasen & Sonographie, CT Abdomen & \\
\hline Lungenmetastasen & Röntgen Thorax in 2 Ebenen & CT Thorax \\
\hline ZNS-Metastasen & Magnetresonanztomographie & \\
\hline
\end{tabular}




\subsection{Klassifikation}

Die Klassifikation ist bei Männern und Frauen identisch, siehe Tabelle 3 und Onkopedia Mammakarzinom der Frau.

Tabelle 3: Klassifikation der Tumorstadien (UICC)

\begin{tabular}{|c|c|c|c|}
\hline $\begin{array}{l}\text { Sta- } \\
\text { dium }\end{array}$ & Primärtumor & Lymphknotenstatus & Fernmetastasen \\
\hline 0 & Tis & NO (keine) & MO \\
\hline \multirow[t]{4}{*}{ I } & Tlmic & NO & MO \\
\hline & Tla $(1-5 \mathrm{~mm})$ & NO & MO \\
\hline & T1b $(6-10 \mathrm{~mm})$ & No & MO \\
\hline & T1c $(11-20 \mathrm{~mm})$ & NO & MO \\
\hline \multirow[t]{2}{*}{ IIA } & T0, T1mic, T1 & $\begin{array}{l}\text { N1 (1-3 LK in der Axilla und/oder der ipsilateralen } \\
\text { Mammaria-Interna-Region) }\end{array}$ & MO \\
\hline & $\mathrm{T} 2(21-50 \mathrm{~mm})$ & NO & MO \\
\hline \multirow[t]{2}{*}{ IIB } & $\mathrm{T} 2$ & N1 & MO \\
\hline & $\mathrm{T} 3(\geq 51 \mathrm{~mm})$ & No & MO \\
\hline \multirow[t]{2}{*}{ IIIA } & T0, T1mic, T1, T2 & N2 (4-9 LK in der Axilla) & MO \\
\hline & T3 & N1 & MO \\
\hline IIIB & $\begin{array}{l}\text { T4 (Infiltration der Brustwand und/ } \\
\text { oder der Haut, und/oder ipsilaterale } \\
\text { Satellitenmetastasen und/oder } \\
\text { inflammatorisches Mammakarzinom) }\end{array}$ & NO - 2 & MO \\
\hline IIIC & alle $\mathrm{T}$ & $\begin{array}{l}\text { N3 ( } \geq 10 \text { LK } n \text { der Axilla und/oder Befall infra- oder } \\
\text { supraklavikulärer LK) }\end{array}$ & MO \\
\hline IV & alle $\mathrm{T}$ & alle $\mathrm{N}$ & $\begin{array}{l}\text { M1 (Metastasen } \\
\text { außerhalb der Brust } \\
\text { und der benachbar- } \\
\text { ten LK-Regionen }\end{array}$ \\
\hline
\end{tabular}

\subsection{Prognostische Faktoren}

In vergleichenden Untersuchungen ist die stadienabhängige Prognose von Männern mit Brustkrebs etwas schlechter als die betroffener Frauen [9, 13, 24, 27, 32, 33, 37], siehe Tabelle 4. Allerdings sind hier das höhere Erkrankungsalter und andere prognostische Faktoren nicht berücksichtigt.

Tabelle 4: Prognose des Mammakarzinoms bei Männern [33]

\begin{tabular}{|l|l|l|}
\hline \multirow{2}{*}{ Stadium } & \multicolumn{2}{|l|}{ 5 Jahres-Überlebensrate (\%) } \\
\cline { 2 - 4 } & Frauen & Männer \\
\hline I & 96 & 78 \\
\hline II & 84 & 66 \\
\hline III & 52 & 39 \\
\hline IV & 24 & 14 \\
\hline
\end{tabular}




\section{Therapie}

\subsection{Therapiestruktur}

Therapieoptionen beim lokal begrenzten Mammakarzinom umfassen die Operation, die Bestrahlung und die medikamentöse Therapie [29]. Viele Empfehlungen für die Behandlung betroffener Männer orientieren an den Empfehlungen für das Mammakarzinom der Frau $[2,3,12]$, weil für diese Patientengruppe eine bessere Evidenzlage vorliegt. Unterschiede finden sich vor allem bei der Radikalität der Operation und bei der endokrinen Therapie. Der Therapiealgorithmus ist in Abbildung 4 dargestellt.

\section{Abbildung 4: Algorithmus für die Primärtherapie}
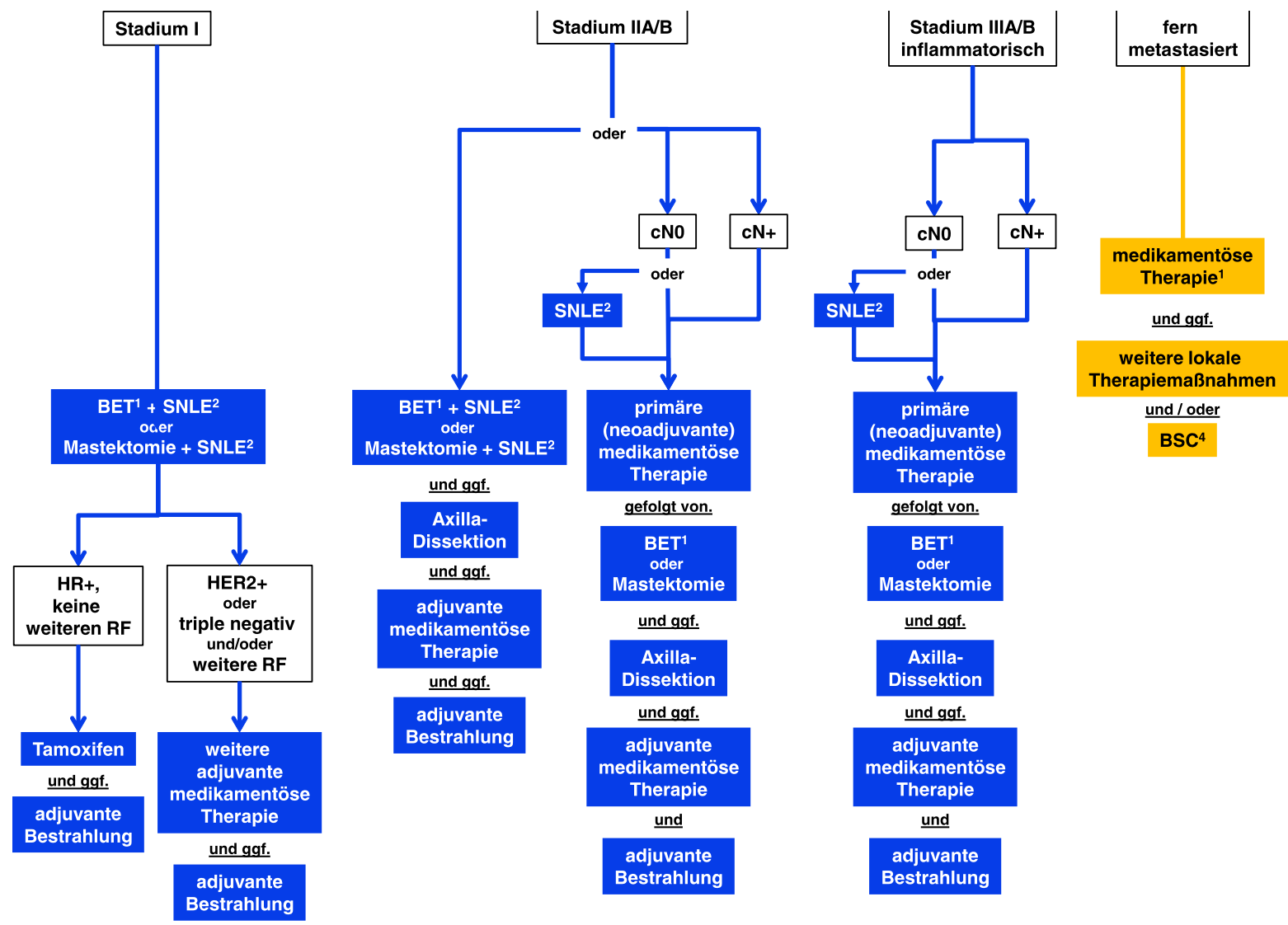

\subsubsection{Lokal begrenzt}

\subsubsection{Operation}

\subsection{Brust}

Bevorzugte Operationstechnik ist die modifizierte radikale Mastektomie. Aber auch die brusterhaltende Operation ist bei geeigneten anatomischen Bedingungen möglich. 


\subsection{Axilla}

Standard zur Evaluation axillärer Lymphknoten bei cNO ist die Sentinel-Lymph-Node-Technik (SLNE) mit gezielter Entfernung und Untersuchung von ein bis drei Lymphknoten, siehe Onkopedia Mammakarzinom der Frau. Retrospektive Studien deuten darauf hin, dass die Sentinel-Lymphknoten-Technik bei Männern ähnlich effektiv wie bei Frauen ist [5, 22]. Die SLNE ist in diesem Stadium der Axilladissektion gleichwertig hinsichtlich der lokalen Kontrolle, aber mit einer geringeren Morbidität belastet. Bei negativem Sentinel-Lymphknoten ist eine Axilladissektion nicht indiziert. Wir empfehlen, analog zum Vorgehen bei den Frauen auch bei positivem Sentinel-Lymphknoten auf die Axilladissektion zu verzichten, wenn folgende Kriterien erfüllt sind:

- nicht mehr als 2 befallene Sentinel-Lymphknoten

- Tumorstadium T1 oder T2

- präoperativ cN0, d. h. kein klinischer Anhalt für einen Befall der Axilla

- intraoperativ kein Hinweis auf Adhärenz der Lymphknoten

- postoperative Bestrahlung durch tangentiale Felder geplant

- adäquate adjuvante medikamentöse Tumortherapie

\subsubsection{Bestrahlung}

Separate prospektive Studien über die Effektivität der Strahlentherapie bei Männern gibt es nicht. In einer retrospektiven Analyse hatten Männer im Stadium III nach adjuvanter Bestrahlung bei Mastektomie ein signifikantes besseres Überleben als Männer ohne Bestrahlung [18]. Bei Männern im Stadium I und II ergab sich durch die zusätzliche Bestrahlung kein prognostischer Unterschied.

Nach brusterhaltender Operation (BET) sollte auch im Stadium I und II die Indikation zur adjuvanten Bestrahlung geprüft werden.

Die Indikationen zur Bestrahlung der Brustwand und der Lymphabflusswege werden im Allgemeinen analog den Kriterien beim Mammakarzinom der Frau bei Zustand nach Mastektomie gestellt. Auch die empfohlenen Dosierungen sind gleich.

\subsubsection{Adjuvante medikamentöse Therapie}

\subsection{Adjuvante endokrine Therapie}

Mehr als 95\% der Mammakarzinome des Mannes exprimieren Östrogen- und/oder Progesteronrezeptoren. Die Kriterien für die Diagnose eines endokrin sensitiven Karzinoms sind bei den beiden Geschlechtern gleich, siehe Onkopedia Mammakarzinom der Frau. Tamoxifen hat sich als endokrine Standardtherapie durchgesetzt, obwohl es keine prospektiv randomisierte Studie im Vergleich zu Placebo oder zu alternativen, hormonablativen Strategien (Orchiektomie, LHRH Analoga) gibt. Tamoxifen verursacht auch bei Männern die typischen Nebenwirkungen, insbesondere reduzierte Libido, Impotenz, Hitzewallungen und Stimmungsschwankungen [1, 25, 33]. Auch das Risiko für venöse Thrombembolien ist erhöht. 
In einer retrospektiven Studie hatten Patienten mit endokriner adjuvanter Therapie ein signifikant besseres Überleben als Patienten ohne adjuvante endokrine Therapie [25]. In einer weiteren retrospektiven Studie aus Deutschland zeigten Männer nach adjuvanter Therapie mit einem Aromatasehemmer eine signifikant schlechtere Prognose als Patienten mit Tamoxifen als adjuvante endokrine Therapie [17].

Bei kombinierter chemoendokriner Therapie sollte die endokrine Behandlung erst nach Abschluss der Chemotherapie begonnen werden.

\subsection{Adjuvante Chemotherapie}

Wegen des im Durchschnitt höheren Krankheitsstadiums bei Erstdiagnose wird die Indikation zur adjuvanten Chemotherapie bei Männern mit Mammakarzinom häufiger als bei Frauen gestellt.

Die einzige prospektive Studie zu diesem Thema wurde mit CMF durchgeführt und zeigte eine 5-Jahres- Überlebensrate von $80 \%$, signifikant besser als die historische Kontrolle [4]. In einer retrospektiven Analyse unter Verwendung von Anthrazyklinen lag die 5-Jahres-Überlebensrate bei $86 \%$ [25]. Im Analogieschluss werden für die Indikation zur adjuvanten Chemotherapie dieselben Kriterien wie beim Mammakarzinom der Frau angewandt, siehe Abbildung 5.

\section{Abbildung 5: Algorithmus für die Chemotherapie}
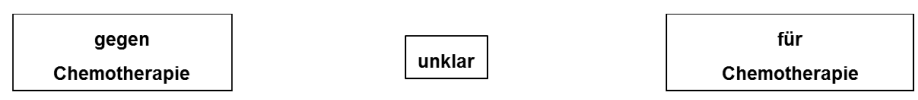

prognostisch

Primärtumor

Nodalstatus

histologischer Grad

Gefäßinfiltration

prädiktiv und prognostisch

HR Status ${ }^{2}$

HER2 Status ${ }^{3}$

molekularer Subtyp

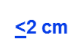

No

G1

fehlt

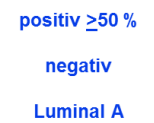

Luminal A
1-3 befallene LK

G2

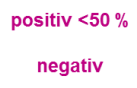

negativ

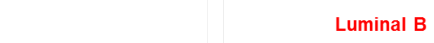

HER2 enriched

Basal like (triple negativ)

$\underline{\text { weitere }}$

Gen-Signatur ${ }^{5}$

Proliferation (Ki67) ${ }^{6}$

UPA / PAI ${ }^{7}$ niedriges Rezidivrisiko

niedrig

niedrig intermediäres Rezidivrisiko

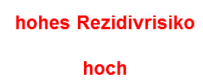

hoch

Legende:

${ }^{1}$ G - Grading;

2 ER - Östrogenrezeptor;

3 HER2 - Human Epidermal growth factor Receptor; HER2 negativ - keine HER2-Überexpression / keine HER2 Genamplifikation; HER2 positiv - HER2 Überexpression und / oder HER2 Genamplifikation;

4 molekular Subtypen - zur Definition;

5 Genexpressionstest - Auswertung der Transkription Prognose-relevanter Gene;

6 Proliferation - Konventionelle Methoden sind der Ki67-Labelling Index und die Auszählung der Mitoserate;

7 UPA / PAl-1 - Urokinase-Type Plasminogen Activator und Plasminogen Activator Inhibitor Type 1 
Die biologischen Parameter der Gen-Signatur, des Proliferationsmarkers Ki67 und von uPA/ PAI sind beim MBC nur in Fallserien untersucht. Bezüglich der Gensignaturen fand sich bei Patienten mit HR-positivem Mammakarzinom eine ähnliche Verteilung wie bei Frauen [28]. Der Wert dieser Marker in der Indikationsstellung einer adjuvanten Chemotherapie ist offen.

Eine wesentliche Einschränkung bei der Indikation und bei der Wahl des Therapieregimes ist die Komorbidät der durchschnittlich älteren Männer.

\subsection{Adjuvante anti - HER2 Therapie}

Eine Überexpression oder Genamplifikation von HER2 wird nur bei 5-10 \% der Männer nachgewiesen [Cardoso]. Daten über die Effektivität gezielter Therapie sind beim virilen Mammakarzinom auf Fallbeispiele aus der palliativen Situation beschränkt. In der adjuvanten Therapie bei Männern wird Trastuzumab in Kombination mit Chemotherapie empfohlen. Bei kardialer Vorbelastung ist die Kombination von Paclitaxel mit Carboplatin zu erwägen.

\subsubsection{Lokal fortgeschrittene Stadien}

\subsubsection{Primäre (neoadjuvante) medikamentöse Therapie}

Bei lokal fortgeschrittenen Karzinomen (Stadien IIIA und B) kann die medikamentöse Therapie auch primär (neoadjuvant, präoperativ) durchgeführt werden mit Applikation von Anthrazyklinen und Taxanen über eine Behandlungsdauer von $\geq 18$ Wochen. Die primäre endokrine Therapie mit Tamoxifen stellt eine Option für Patienten mit ER positivem Tumor dar, bei denen Operation oder Chemotherapie kontraindiziert sind oder abgelehnt werden. Die primäre systemische Chemotherapie ist Teil eines multimodalen Behandlungskonzepts und wird fortgesetzt mit Operation, Bestrahlung sowie endokriner Systemtherapie entsprechend den Indikationen beim lokal begrenzten Mammakarzinom.

\subsubsection{Palliative Therapie}

Auch in dieser Situation gibt es keine eigenständigen Daten aus prospektiv randomisierten Studien bei Männern. Bei Patienten mit ER positivem Mammakarzinom steht die endokrine Therapie mit Tamoxifen an erster Stelle [19]. Effektiv ist auch die Hormonablation (Orchiektomie, LHRH Agonisten \pm Antiandrogen) [26]. In kleinen Studien wurde eine Effektivität von Aromataseinhibitoren beobachtet [16].

Eine Alternative ist der Rezeptorantagonist Fulvestrant [14]. Die Remissionsrate liegen in einer gepoolten Analyse bei $26,1 \%$, das mediane progressionsfreie Überleben bei 5 Monaten [38]. Zur Kombination endokriner Therapie mit gezielten Stoffwechsel-Inhibitoren liegen beim MBC noch keine Daten vor.

Chemotherapie ist auch beim Mammakarzinom des Mannes effektiv, aber mit mehr Nebenwirkungen als die endokrine Therapie belastet. Bei Abwägung von Nutzen und Risiko ist die Chemotherapie indiziert bei Patienten nach Versagen einer endokrinen Therapie und bei ER negativem Karzinom. Sie ist auch indiziert, wenn aufgrund einer fortgeschrittenen, Organ gefährdenden Metastasierung schnell eine Remission erzielt werden soll. 
Bei Patienten mit HER2 positivem Mammakarzinom werden die Anti-HER2-Antikörper Trastuzumab, Pertuzumab und Trastuzumab Emtansin (TDM-1) oder der Kinase-Inhibitor Lapatinib empfohlen.

Bei Mammakarzinomen mit BRCA-Mutationen sind Platinderivate besonders wirksam.

Zur Auswahl geeigneter Substanzen und Kombinationen sowie zur symptomorientierten palliativen Therapie siehe Onkopedia Mammakarzinom der Frau, Kapitel 6.1.4.

\section{Rehabilitation}

Operation, Strahlentherapie und systemische Therapie eines Patienten können zu Therapiefolgestörungen unterschiedlichen Schwergrades führen, die gezielte rehabilitative Maßnahmen im somatischen und psychosozialen Bereich erfordern. Die Patienten sollen über die Möglichkeiten ambulanter und stationärer Rehabilitationsmaßnahmen sowie weiterer Ansprüche, die sich aus dem Sozialrecht ergeben, frühzeitig informiert werden. Hinsichtlich der Rehabilitationsklinik müssen die Wünsche der Patienten berücksichtigt werden. Dennoch sollte eine Empfehlung für eine Klinik mit onkologischem Schwerpunkt abgegeben werden, um einen optimalen Rehabilitationserfolg zu gewährleisten.

\section{Verlaufskontrolle und Nachsorge}

\subsection{Nachsorge}

Ein Ziel der Nachsorge ist die frühzeitige Diagnose von lokoregionären Rezidiven oder eines Zweitkarzinoms mit der Chance einer erneuten Behandlung in kurativer Intention. Dies betrifft beim Mammakarzinom des Mannes insbesondere die jüngeren Patienten und die große Gruppe der Patienten mit bekannter genetischer Prädisposition.

Weitere Ziele sind die Erkennung und Behandlung von Nebenwirkungen der Therapie, und die Fortsetzung der psychosozialen Unterstützung und Beratung. Zeitpunkte und Maßnahmen sind in Tabelle 5 zusammengefasst. Die Empfehlungen sind vom Mammakarzinom der Frau übernehmen.

Eine routinemäßige Suche nach Fernmetastasen ist aufgrund der Unsicherheit der eingesetzten Methoden und der Untersuchungsintervalle bei symptomfreien Patientinnen nicht indiziert. Alle Patienten mit axillärer Lymphadenektomie sollen über die Option der Erkennung, Prophylaxe und Behandlung eines Lymphödems des Arms postoperativ aufgeklärt werden. Die Durchführung einer prophylaktischen Lymphdrainage ist nicht indiziert.

Tabelle 5: Nachsorge nach kurativer Therapie

\begin{tabular}{|c|c|c|c|c|c|c|c|c|c|c|c|c|c|c|c|c|c|c|}
\hline & \multicolumn{18}{|c|}{ Zeitpunkt (Monate) } \\
\hline & 3 & 6 & 9 & 12 & 15 & 18 & 21 & 24 & 27 & 30 & 33 & 36 & 42 & 48 & 54 & 60 & 72 & 84 \\
\hline $\begin{array}{l}\text { Ana- } \\
\text { mnese, } \\
\text { körperli- } \\
\text { che } \\
\text { Untersu- } \\
\text { chung, } \\
\text { Bera- } \\
\text { tung }\end{array}$ & $\mathbf{x}$ & $x$ & $x$ & $x$ & $x$ & $x$ & $x$ & $x$ & $(X)$ & $x$ & (X) & $x$ & $\mathbf{x}$ & $\mathbf{x}$ & $x$ & $x$ & $x$ & $\mathbf{x}$ \\
\hline
\end{tabular}




\begin{tabular}{|c|c|c|c|c|c|c|c|}
\hline & Zeitpunkt (Monate) & & & & & & \\
\hline $\begin{array}{l}\text { Bildge- } \\
\text { bende } \\
\text { Dia- } \\
\text { gnostik } \\
\text { der } \\
\text { Brust }^{1}\end{array}$ & $\mathbf{x}$ & $\mathbf{x}$ & $\mathbf{x}$ & $\mathbf{x}$ & $\mathbf{x}$ & $\mathbf{x}$ & $\mathbf{x}$ \\
\hline
\end{tabular}

Legende:

${ }^{1}$ Standard der apparativen Diagnostik sind Mammographie und Sonographie der betroffenen sowie Mammographie der kontralateralen Brust.

\section{Literatur}

1. Anelli TF, Anelli A, Tran KN et al.: Tamoxifen administration is associated with a high rate of treatment limiting symptoms in male breast cancer patients. Cancer 74: 74-77, 1994. PMID:8004585

2. Arbeitsgemeinschaft Gynäkologische Onkologie: Diagnostik und Therapie primärer und metastasierter Mammakarzinome: Neoadjuvante (Primäre) systemische Therapie, Status Februar 2015. http://www.ago-online.de/fileadmin/downloads/leitlinien/mamma/ maerz2014/de/

2014D_20_Chemotherapie_mit_oder_ohne_zielgerichtete_Substanzen_beim_metastasierten_Mamma

3. AWMF S3 Leitlinie Mammakarzinom der Frau: Diagnostik, Therapie und Nachsorge. http://www.awmf.org/leitlinien/detail/ll/032-045OL.html

4. Bagley CS, Wesley MN, Young RC et al.: Adjuvant chemotherapy in males with cancer of the breast. Am J Clin Oncol 10:55-60, 1987. PMID:3825994

5. Boughey JC, Bedrossian I, Meric-Bernstam F et al.: Comparative analysis of sentinel lymph node operation in male and female breast cancer patients. J Am Coll Surg 203:475-480, 2006. PMID:17000390

6. Brinton LA, Carreon JD, Gierach GL et al.: Etiological factors for male breast cancer in the U.S. Veterans Affairs medical care system database. Breast Cancer Res Treat 119:185-192, 2010. DOI:10.1007/s10549-009-0379-0

7. Brinton LA, Cook MB, McCormack V et al.: Anthropometric and Hormonal Risk Factors for Male Breast Cancer: Male Breast Cancer Pooling Project Results. J Natl Cancer Inst 106: djt465. 2014. DOI:10.1093/jnci/djt465

8. Brinton LA, Key TJ, Kolonel LN et al.: Prediagnostic Sex Steroid Hormones in Relation to Male Breast Cancer Risk. J Clin Oncol 33:2041-2050, 2015. DOI:10.1200/JCO. 2014.59.1602

9. Cardoso F, Bartlett J, Slaets $L$ et al.: Characterization of male breast cancer: First results of the EORTC10085/TBCRC/BIG/NABCG International Male BC Program. SABCS 2014. Cancer Res 75:S6-05, 2015. DOI:10.1158/1538-7445.SABCS14-S6-05

10. Chau A, Jafarian N, Rosa M: Male Breast: Clinical and Imaging Evaluations of benign and malignant entities with histologic correlation. Am J Med 2016: pii: S0002-9343(16)30070-5. DOI:10.1016/j.amjmed.2016.01.009

11. Checkpoint Kinase 2, CHEK2. http://omim.org/entry/604373

12. Coates AS, Winer EP, Goldhirsch A et al.: Tailoring therapies - improving the management of early breast cancer: St Gallen International Expert Consensus on the Primary 
Therapy of Early Breast Cancer 2015. Ann Oncol 26:1533-1546, 2015. DOI:10.1093/ annonc/mdv221

13. Cutili B, Le-Nir CC, Serin D et al.: Male breast cancer: Evolution of treatment and prognostic factors. Analysis of 489 cases. Crit Rev Oncol Hematol 73:246-254, 2010. PMID: 19442535

14. de la Haba Rodriguez JR, Porras Quintela I, Pulido Cortijo G et al.: Fulvestrant in advanced male breast cancer. Ann Oncol 20:1896-1897, 2009. DOI:10.1093/annonc/ mdp413

15. Deutsches Konsortium für hereditären Brust- und Eierstockskrebs; http:// www.mammamia-online.de/MMSpezialBuch.pdf

16. Doyen J, Italiano A, Largillier R, Ferro JM et al.: Aromatase inhibition in male breast cancer patients: biological and clinical implications. Ann Oncol 21:1243-1245, 2010. DOI:10.1093/annonc/mdp450

17. Eggemann $\mathrm{H}$, Ignatov $\mathrm{A}$, Smith $\mathrm{BJ}$ et al.: Adjuvant therapy with tamoxifen compared to aromatase inhibitors for 257 male breast cancer patients. Breast Cancer Res Treat 137:465-470, 2013. DOI:10.1007/s10549-012-2355-3

18. Eggemann $\mathrm{H}$, Ignatov A, Stabenow R et al.: Male Breast Cancer: 20-Year Survival Data for Post-Mastectomy Radiotherapy. Breast Care 2013;8:270-275, 2013. DOI: $10.1159 / 000354122$

19. Eucker J, Kühnl A, Possinger K: Systemische Behandlung des Brustkrebses des Mannes. Zentralbl Chir 2007;132:396-399 DOI:10.1055/s-2007-981267

20. Fentiman IS, Fourquet A, Hortobagyi GN: Male breast cancer. Lancet 2006;367:595-604 PMID:16488803

21. Ferzoco RM, Ruddy KJ: The epidemiology of male breast cancer. Curr Oncol Rep 18:1-9, 2016. DOI:10.1007/s11912-015-0487-4

22. Flynn LW, Park J, Patil SM et al.: Sentinel lymph node biopsy is successful and accurate in male breast carcinoma. J Am Coll Surg 206:616-621, 2008. DOI:10.1016/ j.jamcollsurg.2007.11.005

23. Gesellschaft der epidemiologischen Krebsregister in Deutschland / Robert - Koch Institut: Krebs in Deutschland 2009 - 2010, Häufigkeiten und Trends: Brustdrüse der Frau, 9. Auflage 2013;

24. Giordano SH: A review of the diagnosis and management of male breast cancer. The Oncologist 10:471-479, 2005. DOI:10.1634/theoncologist.10-7-471

25. Giordano $\mathrm{SH}$, Perkins $\mathrm{GH}$, Broglio $\mathrm{K}$ et al.: Adjuvant systemic therapy for male breast carcinoma. Cancer 104:2359-2364, 2005. DOI:10.1002/cncr.21526

26. Giordano SH, Hortobagyi GN: Leuprolide acetate plus aromatase inhibition for male breast cancer. J Clin Oncol 24: e42-43, 2006. DOI:10.1200/JCO.2006.07.2397

27. Goss PE, Reid C, Pintilie M et al.: Male breast carcinoma: a review of 229 patients who presented to the Princess Margaret Hospital during 40 years: 1955 - 1996. Cancer 85:629-639, 1999. PMID:10091736

28. Grenader T, Yerushalmi R, Tokar M et al.: The 21-Gene Recurrence Score Assay (Oncotype DX TM ) in Estrogen Receptor-Positive Male Breast Cancer: Experience in an Israeli Cohort. Oncolgy 87:1-6, 2014. DOI:10.1159/000360793 
29. Korde LA, Zjewski JA, Kamin L et al.: Multidisciplinary meeting on male breast cancer: summary and research recommendations. J Clin Oncol 28:2114-2422, 2010. DOI: 10.1200/JCO.2009.25.5729

30. Ly $D$, Forman $D$, Ferlay J et al.: An international comparison of male and female breast cancer incidence rates. Int J Cancer 132: 1918-1926, 2013. DOI:10.1002/ijc.27841

31. http://www.nicer.org/en/statistics-atlas/cancer-incidence/

32. Nahleh ZA, Srikantiah R, Safa M et al.: Male breast cancer in the Veterans Affairs Population. Cancder 109:1471-1477, 2007. DOI:10.1002/cncr.22589

33. Niederberger $\mathrm{P}$, Bucher $\mathrm{S}$, Aebi S: Männliches Mammakarzinom: Was ist anders? Praxis 105: 147-151, 2016. DOI:10.1024/1661-8157/a002250

34. Silvestri V, Barrowdale D, Mulligan Am et al.: Male breast cancer in BRCA1 and BRCA2 mutation carriers: pathology data from the Consortium of Investigators of Modifiers of BRCA1/2. Breast Cancer Research 18:15- 27, 2016. DOI:10.1186/s13058-016-0671-y

35. http://www.statistik.at/web_de/statistiken/gesundheit/krebserkrankungen/brust/ index.html

36. Wenhui Z, Shuo L, Dabei T et al.: Androgen receptor expression in male breast cancer predicts inferior outcome and poor response to tamoxifen treatment. European Journal of Endocrinology 171: 527-533, 2014. DOI:10.1530/EJE-14-0278

37. $\mathrm{Yu} X F$, Yang $\mathrm{HJ}$, $\mathrm{Yu} Y$ et al.: A prognostic analysis of male breast cancer (MBC) Compared with post-menopausal female breast cancer (FBC). PLoS ONE 10: e0136670, 2015. DOI:10.1371/journal.pone.0136670

38. Zagouri F, Sergentanis TN, Chrysikos D et al.: Fulvestarnt and male breast cancer: a pooled analysis. Breast Cancer Res Treat 149:269-275, 2015. DOI:10.1007/ s10549-014-3240-z

\section{Therapieprotokolle}

- Mammakarzinom - medikamentöse Tumortherapie

\section{Zulassungsstatus}

- Mammakarzinom - Zulassungsstatus von Medikamenten

\section{Links}

www.frauenselbsthilfe.de (eigene Gruppe für Männer mit Brustkrebs)

www.dgho.de/gesellschaft/verein/arbeitskreise/onkologische-rehabilitation

www.mamazone.de 


\section{Anschriften der Verfasser}

Prof. Dr. med. Bernhard Wörmann

Amb. Gesundheitszentrum der Charité

Campus Virchow-Klinikum

Med. Klinik m.S. Hämatologie \& Onkologie

Augustenburger Platz 1

13344 Berlin

Tel: 030450553219

bernhard.woermann@charite.de

Prof. Dr. med. Stefan Aebi

Luzerner Kantonsspital

Departement Medizin

Medizinische Onkologie

CH-6000 Luzern 16

Tel: 004141 205-5860

stefan.aebi@onkologie.ch

Assoc. Prof. PD Dr.med.univ. Marija Balic

Landeskrankenhaus-Universitätskl. Graz

Innere Medizin

Klinische Abt. f. Onkologie

Auenbrugger Platz 15

8036 Graz

Tel: 0043316 385-80556

marija.balic@medunigraz.at

Prof. Dr. med. Thomas Decker

Onkologie Ravensburg

Elisabethen Str. 19

88212 Ravensburg

Tel: 07513661970

Fax: 0751366197

decker@onkonet.eu

Prof. Dr. Tanja Fehm

Universitätsklinikum Düsseldorf

Frauenklinik

Moorenstr. 5

40225 Düsseldorf

Tel: $0211811-7501$

Tanja.Fehm@med.uni-duesseldorf.de 
Prim. Univ.-Prof. Dr. Richard Greil

Landeskrankenhaus Salzburg

Universitätsklinik f. Innere Medizin III

Onkologisches Zentrum

Müllner Hauptstr. 48

A-5020 Salzburg

Tel: 0043662 4482-2879

Fax: 0043662 4482-2898

r.greil@salk.at

Prof. Dr. med. Nadia Harbeck

Universität München Campus Innenstadt

Frauenkliniken Maistr.

Brustzentrum + Onkologische Tagesklinik

Marchionistr. 15

81377 München

Tel: 089 7095-7581

nadia.harbeck@med.uni-muenchen.de

\section{Prof. Dr. med. Barbara Krug}

Uniklinik Köln

Institut für diagnostische und interventionelle Radiologie

Kerpener Straße 62

50931 Köln

Tel: 022147896062

barbara.krug@uk-koeln.de

\section{Dr. med. Friedrich Overkamp}

OncoConsult. Hamburg $\mathrm{GmbH}$

Am Kaiserkai 1

20457 Hamburg

Tel: 01726117603

overkamp@oncoconsult.hamburg

Prof. Dr. med. Oliver Rick

Klinik Reinhardshöhe

Hämatologie/Onkologie

Quellenstr. 8-12

34537 Bad Wildungen

Tel: $05621705-154$

Fax: 05621 705-450

oliver.rick@klinik-reinhardshoehe.de

Prof. Dr. Frederik Wenz

Universitätsmedizin Mannheim

Klinikdirektor - Klinik für Strahlentherapie und Radioonkologie

Theodor-Kutzer-Ufer 1-3

68167 Mannheim

Tel: 0621 383-4960

frederik.wenz@umm.de 
Prof. Dr. med. Diana Lüftner

Charité Campus Benjamin Franklin

Med. Klinik m.S. Hämatologie,

Onkologie und Tumorimmunologie

Hindenburgdamm 30

12200 Berlin

Tel: $030450-513524$

Fax: $030450-513919$

diana.lueftner@charite.de

\section{Erklärungen zu möglichen Interessenkonflikten}

nach den Regeln der DGHO Deutsche Gesellschaft für Hämatologie und Medizinische Onkologie und den Empfehlungen der AWMF (Version vom 23. April 2010) und internationalen Empfehlungen. 